\title{
Hierarchical Control Strategy for an Integrated Wastewater Treatment Plant
}

\author{
Xi Zhang ${ }^{*, \star}$ Karlene A. Hoo ${ }^{* *}$ \\ * Department of Chemical Engineering, Texas Tech University, Lubbock, TX \\ 79409 USA (e-mail: xi.zhang@ttu.edu). \\ ** Department of Chemical Engineering, Texas Tech University, Lubbock, TX \\ 79409 USA (e-mail: karlene.hoo@ttu.edu)
}

\begin{abstract}
This paper presents the design of a hierarchical control system consisting of a model based centralized supervisory controller and a group of embedded controllers for a set of integrated biological reactors, which is a part of NASA's advanced regenerative wastewater treatment system. A first principle model developed by [Zhang and Hoo, 2007] is used to design the centralized model predictive controller to regulate the performance of the integrated biological reactors for on-supply \& ondemand operations simultaneously. The embedded control is implemented as safety mode to guarantee the process throughput performance in case of malfunction or the loss of communication with the higher level supervisory controller. The control design is implemented and tested on a testbed that is $1 / 20^{t h}$ the scale of the actual biological reactors.
\end{abstract}

\section{INTRODUCTION}

The coupled biological reactors used in this study were designed by NASA Johnson Space Center (JSC) as part of a regenerative biological wastewater treatment plant for longterm human space missions to the moon and Mars ([Muirhead et al., 2003]). Unlike current 7-day shuttle missions, the extended period of time for future exploration missions will be over one thousand days. Thus, such missions will require robust technologies that can operate at extreme conditions (e.g., microgravity, radiation) and with minimal human intervention (i.e. autonomous).

The primary purpose of this work is to investigate robust and autonomous control designs for regenerative systems. The testbed selected is the wastewater treatment pre-processing system whose purpose is to process $22 \mathrm{~L} /$ day of raw feed water so that the downstream processing system can provide potable water for the mission crew. The major unit operations in the preprocessing stage are a packed bed reactor and a pair of tubular reactors. These reactors support two types of microorganisms - nitrifying and denitrifying bacteria. The biological reactors are linked by a recycle loop, hence propagation of certain types of disturbances may lead to total system failure. As with most biological systems, the system's response is slow, highly interactive, multivariable, and constrained. The choice of the control strategy and configuration must account for these difficult issues.

Model predictive control (MPC) is one type of control strategy that has been applied successfully in the chemical and petrochemical industries because of its ability to address multivariable and constrained control problems [Brosilow and Joseph, 2002]. However, even with advanced and complex control strategies there is always the opportunity for controller malfunctions leading to catastrophic system failure. In contrast, even though embedded control has been applied widely and reliably in robotics and mechatronics, as a result of its compact

\footnotetext{
^ This work was supported by a NASA grant and an TTU AT\&T Fellowship.
}

structure, low dependency on external device and high integrity on software and hardware, embedded control hasn't made the same inroads in process control applications.

In this work, a hierarchical control configuration is proposed to control the coupled biological reactors. This hierarchical configuration consists of both a model-based supervisory level controller and embedded controllers. The control strategy relies on the embedded controllers to maintain a minimum level of regulation whenever the centralized supervisory controller fails. To assess the benefits of this hierarchical control configuration, it is implemented on the wastewater treatment testbed at different test scenarios

The paper is organized as follows. Section 2 introduces the testbed and a first-principles kinetic-based model of the testbed. In section 3, a description of the hierarchical control configuration is provided and in section 4, the design and implementation of the embedded controllers are presented. In section 5, the centralized model-based supervisory controller is designed. All the tuning parameters are pre-selected using the simulated results of the first-principles model. The closed-loop performance results of applying the hierarchical control configuration to the testbed under various test scenarios are presented, analyzed and compared in section 6. Lastly, section 7 summarizes the work presented and discusses future research.

\section{COUPLED BIOLOGICAL REACTORS}

The entire wastewater treatment plant is designed to treat yellow water (human urine plus urine flush) and grey water, which is defined as wastewater that is generated from shower, hand washing, and humidity condensate. Two main species in this waste stream require treatment, total organic carbon (TOC) and ammonium ion $\left(\mathrm{NH}_{4}^{+}\right)$[Verostko et al., 2004]. As illustrated in Fig. 1, the treatment system consists of pre- and post- processing stages. In the former there are two coupled biological reactors - a packed bed, denitrification reactor (PB) that operates anaerobically, and a pair of nitrification reactors (TR) that 
operate aerobically. The main purpose of the pre-processing stage is to reduce TOC by denitrification in the PB and to remove ammonium ion by nitrification in the TR. This design is attractive because in theory, the reduction of TOC and removal of ammonium ion can be achieved without the addition of other chemicals. Further, the nitrogen gas released can be used for other applications such as natural plant growth.

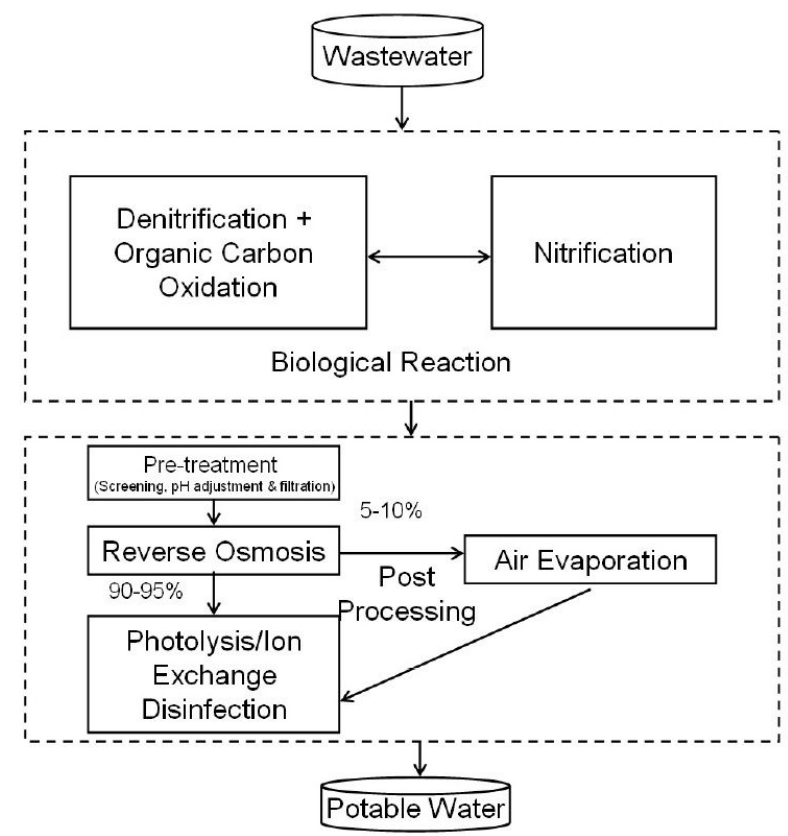

Fig. 1. Schematic of the process.

\subsection{Experimental setup}

The testbed used in this study is $1 / 20^{t h}$ the size of the actual NASA designed wastewater treatment system. It is instrumented as shown schematically in Fig. 2. The feed tank supplies grey and yellow water continuously using a peristaltic pump at a rate $Q_{0} \mathrm{~mL} / \mathrm{min}$, which is set by the mission requirement (onsupply constraint). Combined with the flow from the recycle line, the feed is delivered to the PB. In the PB, the pressure is regulated around $172 \mathrm{kPa}$ to keep nitrogen, a by-product of the denitrification, dissolved in the liquid phase. The tubular reactors are made of high-density polyethylene (HDPE). A peristaltic pump delivers the effluent of the $\mathrm{PB}, Q_{2} \mathrm{~mL} / \mathrm{min}$, that is mixed with a dedicated air stream to the TR. The TR operates at atmospheric pressure. Finally, a gas-liquid separator (GLS) is used to ensure that only a liquid phase is recirculated. The purpose of the liquid recycle line is to improve the efficiency of the waste treatment. There is a minimum flow rate constraint required by the post-processing stage. Thus, the post-processing stage imposes an on-demand constraint on the pre-processing stage.

The microorganism obtain energy and grow by transferring electrons from donor compounds to acceptor compounds [Rittman and McCarty, 2001]. Here, the half reactions for TOC and $\mathrm{NH}_{4}^{+}$in the $\mathrm{PB}$ and the TR respectively are [Jackson],

$$
\begin{aligned}
0.0025 \mathrm{TOC}+0.33 \mathrm{H}_{2} \mathrm{O} & \rightarrow 0.2 \mathrm{CO}_{2}+0.02 \mathrm{NH}_{4}^{+}+\mathrm{e}^{-} \\
0.125 \mathrm{NH}_{4}^{+}+0.375 \mathrm{H}_{2} \mathrm{O} & \rightarrow 0.125 \mathrm{NO}_{3}^{-}+1.25 \mathrm{H}^{+}+\mathrm{e}^{-}
\end{aligned}
$$

Most biological reactions are enzyme-catalyzed. If the metabolic processes of the bacteria can be maintained at an optimally active level, efficient treatment of the wastewater can be achieved.

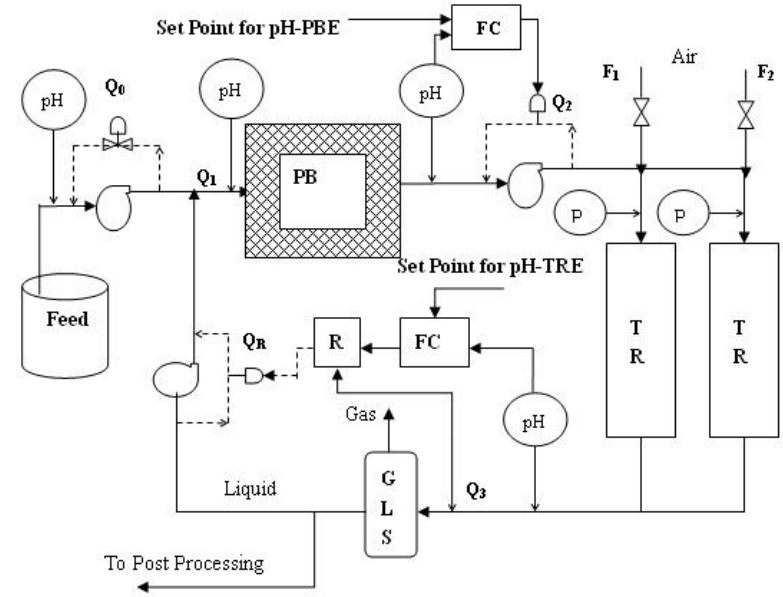

Fig. 2. Schematic of the instrumented testbed.

However, enzyme-catalyzed reactions are dependent on $\mathrm{pH}$, pressure and temperature, which affect the activity of the enzyme. In this system, the temperature is regulated to $25^{\circ} \mathrm{C}$. From a stoichiometric analysis and experimental observations, it is found that the $\mathrm{pH}$ of the $\mathrm{PB}$ reactor will increase by approximately 0.5 units for one unit of $\mathrm{H}^{+}$consumed and one unit of $\mathrm{NH}_{4}^{+}$generated. In contrast, the $\mathrm{pH}$ of the TR reactor will decrease by almost 2 units for the generation of unit of $\mathrm{H}^{+}$ and the consumption of one unit of $\mathrm{NH}_{4}^{+}$. From experiments, the $\mathrm{pH}$ range for each measurement point is listed in Table 1. The effluent $\mathrm{pH}$ is assumed to be the standard $\mathrm{pH}$ for bacteria growth.

Table 1. $\mathrm{pH}$ at different measurement locations.

\begin{tabular}{ccccc}
$\mathrm{pH}$ & Feed & PB Inlet & PB Effluent & TR Effluent \\
\hline & & & & \\
Max. & 9.2 & 8.2 & 8.8 & 7.4 \\
Min. & 8.2 & 7.0 & 7.8 & 6.2 \\
\hline
\end{tabular}

An automated Real-Time Monitoring System (RTMS) is designed and implemented to collect the experimental data (see Fig. 2, including $4 \mathrm{pH}$ electrodes and 2 pressure transducers for the injection of air). All the instrumentation are connected to a National Instruments (NI) data acquisition (DAQ, PCI-6031E) card that is part of the dedicated data collection system. A realtime visual interface also is designed using NI's LabVIEW ( $\mathrm{v}$ 8.0) to display the real-time data and to provide a visual monitor of the system's performance.

\subsection{Computational model development}

The biological wastewater treatment processes can accommodate both film and suspension (bulk) growth. The following is taken from [Zhang and Hoo, 2007]. The variables and parameters are listed in Table 2. It should be noted that superscripts ${ }^{P}$ and ${ }^{T}$ represent $\mathrm{PB}$ and TR reactors, respectively; while subscripts $_{b}$ and $f$ denote bulk or film, respectively.

$P B$ reactor The model equations that describe the $\mathrm{PB}$ reactor are given by (1) to (5). A species balance on the substrate, $S_{b}^{P}$, can be derived by considering the inlet and outlet flow rates of the substrate concentration and consumption of the substrate by the biomass in the bulk and film phases, [Zhang et al., 2006, Rittman and McCarty, 2001]. 
Table 2. Design and operating parameters ([Rittman and McCarty, 2001]).

\begin{tabular}{|l||l||c||c||}
\multicolumn{1}{c}{ Definition } & \multicolumn{1}{c}{ Symbol } & \multicolumn{1}{c}{ PB } \\
\hline$K \mathrm{mgSub} / \mathrm{mgVSS}$-day & Max. spec. sub. rate & 0.0324 & 2.2 \\
$K_{s} \mathrm{mg} / \mathrm{L}$ & Saturation const. & 15.3152 & 24.4330 \\
$Y \mathrm{mgVSS} / \mathrm{mgSub}$ & Cell mass/sub yield & 0.8 & 0.2 \\
$b \mathrm{day}^{-1}$ & Cell death coeff. & 0.01 & 0.36 \\
$b_{s} \mathrm{day}^{-1}$ & Biofilm shear coeff. & 0.01 & 0.0002 \\
$D_{f} \mathrm{~cm}^{2} /$ day & Biofilm diff. & 1.87 & 1.87 \\
$k_{f} \mathrm{~cm} / \mathrm{day}$ & Mass transfer coeff. & 10.3613 & 29.2378 \\
$V_{v} \mathrm{~L}$ & Reactor void vol & 2.2 & 0.24 \\
$A \mathrm{~cm}^{2}$ & Biofilm surf. area & 17703 & 3039 \\
$Q \mathrm{~L} / \mathrm{day}$ & Feed flow rate & 21.6 & 21.6 \\
$S_{0} \mathrm{mg} / \mathrm{L}$ & Feed sub. conc. & 369.82 & 208.64 \\
$S_{b^{i}} \mathrm{mg} / \mathrm{L}$ & Init. bulk sub. & 10 & 100 \\
$X_{b^{i}} \mathrm{mgVSS} / \mathrm{L}$ & Init. bulk biomass & 0.1 & $\mathrm{NA}$ \\
$L_{f^{i}} \mathrm{~mm}$ & Init. film thick & 0.02 & 0.01 \\
$X_{f^{i}} \mathrm{mgVSS} / \mathrm{L}$ & Init. film biomass & 2000 & 100 \\
$S_{f^{i}} \mathrm{mg} / \mathrm{L}$ & Init. film sub. & 280 & 2 \\
\hline
\end{tabular}

$$
\begin{array}{r}
\frac{d S_{b}^{P}}{d t}=\frac{Q^{P}}{V_{v}^{P}}\left(S_{0}^{P}-S_{b}^{P}\right)-\left(\frac{k^{P} S_{b}^{P}}{K_{s}^{P}}+S_{b}^{P}\right) X_{b}^{P}-k_{f}^{P} \frac{A^{P}}{V_{v}^{P}} \\
\times\left(S_{b}^{P}-\left.S_{f}^{P}\right|_{z=L_{f}^{P}}\right)
\end{array}
$$

It is assumed that the rate of $S_{b}^{P}$ consumption can be represented by Monod kinetics and that mass transport from the bulk to the film is suitably described by Fick's first law. A species balance on the bulk and film biomass includes growth kinetics, cell death rate, and the effect of shearing.

$$
\frac{d X_{b}^{P}}{d t}=-\frac{Q^{P}}{V_{v}^{P}} X_{b}^{P}+\left(\frac{Y^{P} k^{P} S_{b}^{P}}{K_{s}^{P}+S_{b}^{P}}-b^{P}\right) X_{b}^{P}+\int_{0}^{L_{f}^{P}} \frac{A^{P}}{V_{v}^{P}} b_{s}^{P} X_{f}^{P} d z
$$

The diffusion of the substrate to the biomass growing in the film is described by Fick's second law. Further, the utilization of the substrate by the film biomass follows Monod kinetics.

$$
\begin{gathered}
\frac{\partial S_{f}^{P}}{\partial t}=D_{f}^{P} \frac{\partial^{2} S_{f}^{P}}{\partial z^{2}}-\frac{k^{P} S_{f}^{P}}{K_{s}^{P}+S_{f}^{P}} X_{f}^{P} \\
\int_{0}^{L_{f}^{P}} \frac{\partial X_{f}^{P}}{\partial t} d z=\int_{0}^{L_{f}^{P}}\left(\frac{Y^{P} k^{P} S_{f}^{P}}{K_{s}^{P}+S_{f}^{P}}-b^{P}-b_{s}^{P}\right) X_{f}^{P} d z
\end{gathered}
$$

The change in the length of the biofilm, $L_{f}^{P}$, is a sum of the biomass film growth,

$$
\frac{d L_{f}^{P}}{d t}=\int_{0}^{L_{f}^{P}}\left(\frac{Y^{P} k^{P} S_{f}^{P}}{K_{s}^{P}+S_{f}^{P}}-b^{P}-b_{s}^{P}\right) d z
$$

The boundary conditions for the PB reactor are,

$$
\begin{aligned}
& \left.\frac{\partial X_{f}^{P}}{\partial z}\right|_{z=0}=\left.0 \quad \frac{\partial S_{f}^{P}}{\partial z}\right|_{z=0}=0 \\
& \left.D_{f}^{P} \frac{\partial S_{f}^{P}}{\partial z}\right|_{z=L_{f}^{P}}=k_{f}^{P}\left(S_{b}^{P}-\left.S_{f}^{P}\right|_{z=L_{f}^{P}}\right)
\end{aligned}
$$

TR reactor The hydraulic retention time (HRT) in the TR is very short, hence only biomass film growth occurs in the TR. The air rate to the TR is in excess (80:1) when compared to the rate of the feed stream. Applying a similar approach as that used for the PB reactor gives,

$$
\begin{aligned}
\frac{d S_{b}^{T}}{d t} & =\frac{Q^{T}}{V_{v}^{T}}\left(S_{0}^{T}-S_{b}^{T}\right)-k_{f}^{T} \frac{A^{T}}{V_{v}^{T}}\left(S_{b}^{T}-\left.S_{f}^{T}\right|_{z=L_{f}^{T}}\right) \\
\frac{d X_{f}^{T}}{d t} & =\int_{0}^{L_{f}^{T}}\left(\frac{Y^{T} k^{T} S_{f}^{T}}{K_{s}^{T}+S_{f}^{T}}-b^{T}\right) d z \frac{X_{f}^{T}}{L_{f}^{T}}-\frac{A^{T}}{V_{v}^{T}} b_{s}^{T} X_{f}^{T} L_{f}^{T} \\
\frac{\partial S_{f}^{T}}{\partial t} & =D_{f}^{T} \frac{\partial^{2} S_{f}^{T}}{\partial z^{2}}-\left(\frac{k^{T} S_{f}^{T}}{K_{s}^{T}+S_{f}^{T}}\right) X_{f}^{T} \\
\frac{d L_{f}^{T}}{d t}= & \int_{0}^{L_{f}^{T}}\left(\frac{Y^{T} k^{T} S_{f}^{T}}{K_{S}^{T}+S_{f}^{T}}-b^{T}-b_{s}^{T}\right) d z
\end{aligned}
$$

Suitable boundary conditions for the TR are given by,

$$
\begin{aligned}
& \left.\frac{\partial S_{f}^{T}}{\partial z}\right|_{z=0}=0 \\
& \left.D_{f}^{T} \frac{\partial S_{f}^{T}}{\partial z}\right|_{z=L_{f}^{T}}=k_{f}^{T}\left(S_{b}^{T}-\left.S_{f}^{T}\right|_{z=L_{f}^{T}}\right)
\end{aligned}
$$

Model solution \& validation The numerical solution to the above system of partial differential equations is found using a variable space grid finite difference method. The steady state values of the nonlinear model are validated against the testbed data at different inlet feed flowrates and feed compositions [Zhang and Hoo, 2007]. Although quantitatively there are differences between the model's predictions and the testbed data for some conditions, the model is satisfactory qualitatively at predicting the trends in the system's performance. The steady state solutions for $S_{b}^{P}$ and $s_{b}^{T}$ at the nominal operating condition will be used as the set points in the design of the regulatory controllers $\left(S_{b}^{P}=55.6 \mathrm{mg} / \mathrm{L}\right.$ and $\left.S_{b}^{T}=96.9 \mathrm{mg} / \mathrm{L}\right)$.

\section{HIERARCHICAL CONTROL DESIGN WITH INTEGRATED FINITE STATE MACHINE}

A hierarchical control architecture that achieves a limited degree of autonomy at different levels of the hierarchy is described by [Antsaklis et al., 1991]. The multiple-level strategy proposes to achieve a limited degree of autonomy by the integration of intelligence and robustness. The intelligence is embodied in the global and prediction-based behavior of the centralized supervisory controller, while the robustness is obtained by the use of distributed regulators. The wastewater treatment process in this work provides an opportunity to test this hierarchical control architecture. Specifically, a validated firstprinciples model will be used to develop the intelligence in the centralized supervisory controller at one level and distributed embedded controllers will be used at the regulatory level. A schematic of this control architecture is shown in Fig. 3.

This hierarchical control architecture is both a functional architecture and a structural arrangement. Two distributed singleloop feedback controllers are configured at the execution level using embedded control technology. The embedded controllers permit continuous independent regulation (safety mode) should the connectivity with the other control levels be disconnected. Embedded technology with "direct coding on a chip" provides greater reliability when compared to standard implementation because its implementation combines both hardware and software [Bleris et al., 2006]. Also, the compact structure of an embedded controller allows for local instrumentation which 


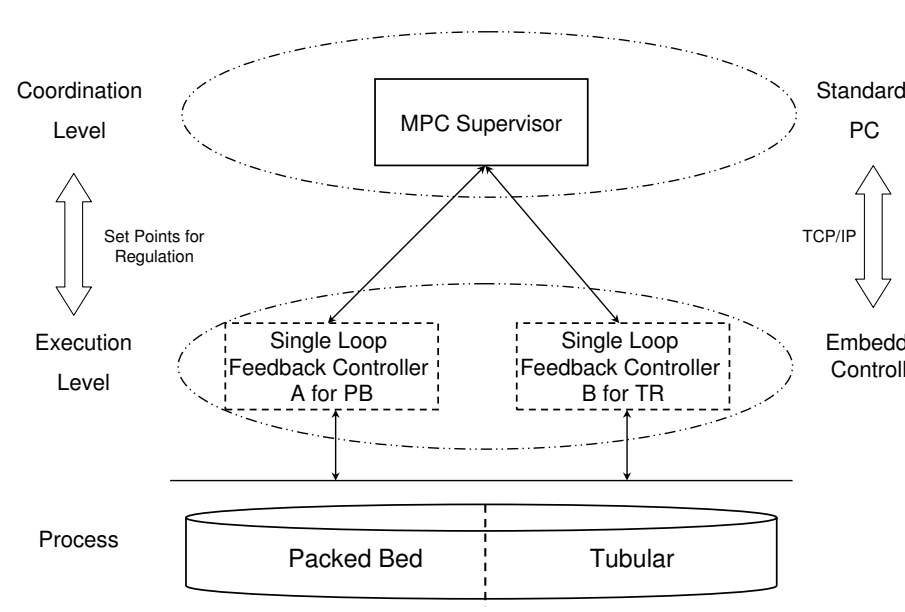

Fig. 3. Schematic of the hierarchical control architecture.

reduces the communication lag and promotes a distributed setup arrangement. However, the scope of an embedded controller is local, which leads to limited regulation performance because issues such as coupling effects are not included.

The intelligent centralized supervisory control system is designed to coordinate the regulation of the two reactors. The supervisory controller uses an MPC algorithm based on a model $(\S 2.2)$ of the integrated wastewater treatment plant and is capable of optimizing the throughput performance of the process globally.

\subsection{Dual Modes and the Finite State Machine}

There are two modes for the hierarchical control configuration, the normal mode and the safety mode. The strategy for the normal mode is that the supervisory system is responsible for updates of the set points of the local controllers. Once the supervisory controller malfunctions or the communication between the levels is lost, the safety mode is triggered which allows the embedded controllers to use default control laws and set points to continue regulating the process. A finite state machine (automata), (Fig. 4), which is event driven, will be used to achieve the mode shift [Bemporad and Morari, 1999, Lemmon et al., 1999].

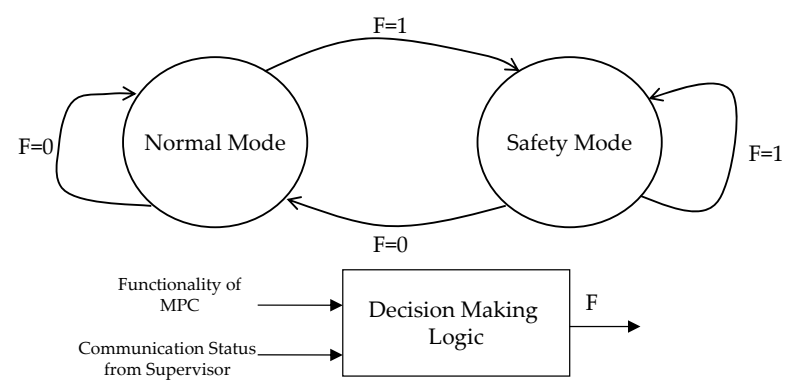

Fig. 4. Finite state machine (automata).

The boolean signal $\mathrm{F}$ indicates the functioning pattern of the hierarchical control system and is used as the event for the automata. The boolean signal $\mathrm{F}$ remains in the 0 state as long as the decision making logics detect no malfunction of the centralized controller and no loss of communication between the control levels. If either of these errors appears on the input side of the decision making block, $F$ will switch to the 1 state and trigger the safety mode operation. The safety mode design places the embedded controllers into a non-supervisory control mode. Only when both errors are fixed, will the boolean signal $\mathrm{F}$ switch to the 0 state and allow communication between the two control levels.

\section{EMBEDDED CONTROL}

The algorithm for the embedded controller uses a feedback dproportional-only control law. The variables to be controlled are $S_{b}^{P}$ and $S_{b}^{T}$. Empirical relationships can be developed to estimate $S_{b}^{p}$ and $S_{b}^{T}$ using the real-time $\mathrm{pH}$ measurements. The manipulated variables are $Q_{R}\left(\right.$ control $\left.S_{b}^{P}\right)$ and $Q_{2}\left(\right.$ control $\left.S_{b}^{T}\right)$. A relative gain array calculation supports this loop pairing [Ogunnaike and Ray, 1994].

The main challenges for process regulation are the disturbances in the raw feed tank concentration and the operation of the system itself (inefficient metabolism, poor growth rate, shedding, fouling, stiction, etc.). In this system, concentration disturbances in the raw feed have the largest impact on the performance of the coupled reactors.

Figure 5 shows the closed-loop control performance of the coupled reactor system for a $10 \%$ increase in both TOC and $\mathrm{NH}_{4}^{+}$concentrations introduced at time 0 in the raw feed tank. The controller gains are -1.0 and $20.0 \mathrm{~mL} / \mathrm{min}$ for the $S_{b}^{P}: Q_{R}$ and $S_{b}^{T}: \mathrm{Q}_{2}$ loops, respectively. The time constant of the PB reactor is about 140 minutes while that of the TR reactor is approximately 40 minutes. The control actions are satisfactory and there is no violation of any physical limits [Zhang and Hoo, 2007]. The overshoot is about $1 \%$ for both the effluent of the $\mathrm{PB}$ (PBE) and TR (TRE). In the case of the PB reactor, the settling time is about 350 minutes after the introduction of the feed composition disturbance. When compared to the settling time of the TR reactor, the settling time of the $\mathrm{PB}$ reactor is $42 \%$ smaller.
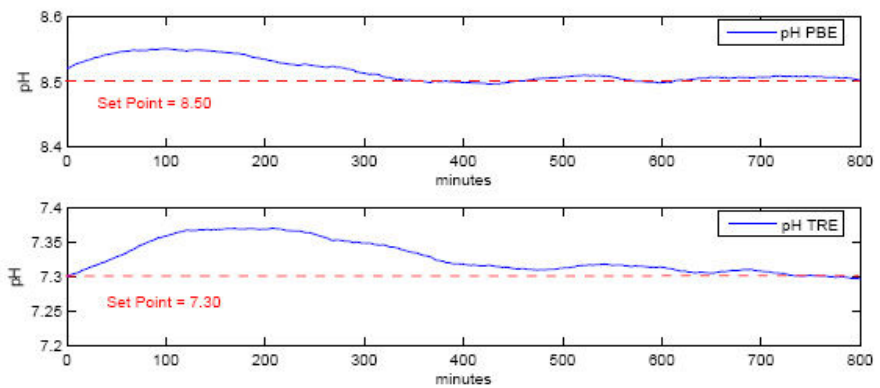

Fig. 5. Closed-loop performance with single-loop controllers.

For the expected range of unmeasured disturbances, the fixed parameter feedback controllers can regulate the system to their desired set points. Thus, the proportional-only control law also will be used as the default control law in the embedded controllers.

\section{MODEL PREDICTIVE SUPERVISORY CONTROLLER}

The quality of a model predictive controller (MPC) is dependent on the type of model used. The cases successfully demonstrated in industry all employ linear step response (input/output) models to control nonlinear processes. In theory, a nonlinear MPC controller can be designed however, closed-loop stability is difficult to establish and convergence of the optimization 
formulation, which is the basic underpinning of the MPC solution, is not guaranteed. Since the objective of the wastewater treatment system is to operate at the designed conditions (reject disturbances) it is not unreasonable to develop an MPC formulation based on an approximate linear perturbation model derived from the validated nonlinear first-principles model.

\subsection{Linear Model and System Analysis}

Consider the following general form of a nonlinear model,

$$
\begin{aligned}
\dot{\mathbf{x}} & =\mathbf{f}(\mathbf{x}, \mathbf{u}) \\
\mathbf{z} & =\mathbf{g}(\mathbf{x})
\end{aligned}
$$

where $\mathbf{f}, \mathbf{g}$ are real-valued vector functions and $\mathbf{x} \in R^{\mathrm{n}}$ and $\mathbf{u} \in R^{\mathrm{m}}$. In the wastewater system, $\mathrm{n}=9$ and $\mathrm{m}=2$. Applying a Taylor series approximation about the designed stable values $\left(\mathbf{x}_{e}, \mathbf{u}_{e}\right)$ gives a linear time-invariant (LTI) model,

$$
\begin{aligned}
\dot{\boldsymbol{\xi}} & =\mathbf{A} \boldsymbol{\xi}(t)+\mathbf{B m}(t) \\
\Delta \mathbf{y} & =\mathbf{C} \boldsymbol{\xi}(t)
\end{aligned}
$$

where $\boldsymbol{\xi}=\mathbf{x}-\mathbf{x}_{e} \in R^{\mathrm{n}}, \mathbf{m}=\mathbf{u}-\mathbf{u}_{e} \in R^{\mathrm{m}}$, and $\Delta \mathbf{y}=\mathbf{y}-\mathbf{y}_{e} \in R^{\mathrm{m}}$, $\mathbf{A} \in R^{\mathrm{n} \times \mathrm{n}}$ is the dynamic system matrix, $\mathbf{B} \in R^{\mathrm{n} \times \mathrm{m}}$ is the input transfer matrix, and $\mathbf{C} \in R^{\mathrm{m} \times \mathrm{n}}$ is the measurement matrix.

The number of manipulated variables represents the number of control degrees of freedom. The number and location of the manipulated variables are very important for effective feedback control. In the case of state controllability [Kailath, 1980], the LTI system is found to be fully state controllable. There are seven state variables. Using the real-time measurements and knowledge of the stoichiometric reactions in the PB and TR reactors, estimates of the state variables can be obtained. The LTI system is found to be fully state observable [Kailath, 1980].

\subsection{MPC design}

The MPC design will employ the same controlled and manipulated variables as the embedded controllers. Under normal operation, the set points of the manipulated variables are provided to the embedded controllers by the MPC. Thus, for local controllers, their set points are updated at every control interval. The value of $Q_{R}$ will be determined from the approximate linear model's estimate of $R$ and the real-time measurement of $\mathrm{Q}_{3}$. The MPC design parameters are a sampling period of 5 minutes, a control horizon of 3 sampling intervals, and a prediction horizon of 40 sampling periods. Table 3 gives the limits and weights on the controller variables.

Two different types of disturbances will be used to evaluate the performance of the MPC design. Here the RFT's (Raw Feed Tank) TOC disturbance is estimated from a measurement of the RFT's pH measurement, but the RFT of $\mathrm{NH}_{4}^{+}$disturbance is assumed unmeasured, as illustrated in Fig. 6.

Table 3. MPC design.

\begin{tabular}{cccccc} 
Variables & Min & Max & Max Decrease & Max Increase & Weights \\
& & & & & \\
$\Delta R$ & -0.4 & 0.05 & -0.05 & 0.01 & 0.5 \\
$\Delta Q_{2} \mathrm{~mL} / \mathrm{min}$ & -7.5 & 7.5 & -0.14 & 0.14 & 0.5 \\
\hline
\end{tabular}

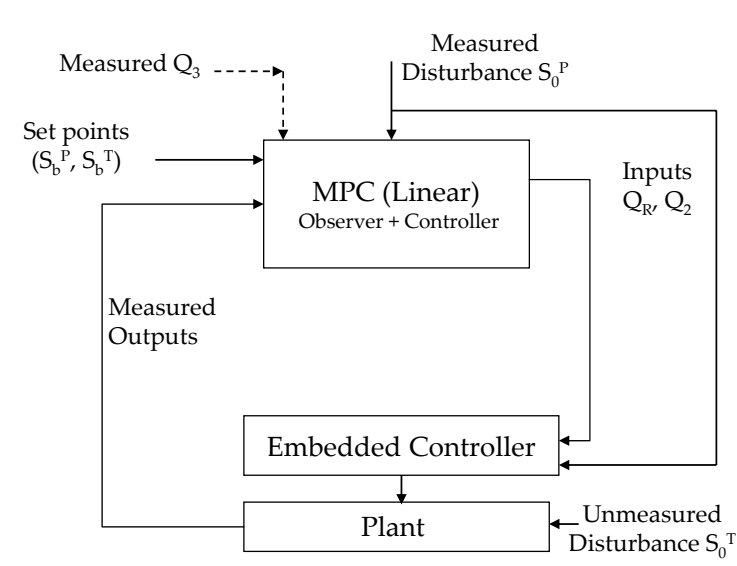

Fig. 6. MPC and embedded controller 2-level structure.

\subsection{State Observer Design}

Of the states that describe this multiple-input multiple-output system only two of the seven states are measurable in realtime. To provide estimates of the unmeasurable states from the available measurements a discrete state estimator in the following form is developed and implemented using Matlab (Natick, MA) toolbox,

$$
\begin{aligned}
\mathbf{x}(k+1) & =\mathbf{A x}(k)+\mathbf{B}_{u} \mathbf{u}(k)+\mathbf{B}_{v} \mathbf{v}(k) \\
\mathbf{y}_{m}(k) & =\mathbf{C}_{m} \mathbf{x}(k)+\mathbf{D}_{v m} \mathbf{v}(k)
\end{aligned}
$$

where $\mathbf{x}(k) \in R^{n}$ is the state vector, $\mathbf{y}_{m}(k) \in R^{p}$ are the measured outputs, $\mathbf{v}(k) \in R^{r}$ are the disturbances, $\mathbf{u}(k) \in R^{m}$ are the manipulated inputs, and $k$ is the sample time. Matrices $\mathbf{C}_{m}, \mathbf{A}$, $\mathbf{B}_{u}$ and $\mathbf{D}_{v m}$ are real-valued and of the appropriate dimensions. The estimator's design includes three parts: output prediction, measurement update, and states' estimate at the next sample time.

$$
\begin{aligned}
\hat{\mathbf{y}}_{m}(k \mid k-1) & =\mathbf{C}_{m} \hat{\mathbf{x}}(k \mid k-1)+\mathbf{D}_{v m} v(k) \\
\hat{\mathbf{x}}(k \mid k) & =\hat{\mathbf{x}}(k \mid k-1)+\mathbf{M}\left(\mathbf{y}_{m}(k)-\hat{\mathbf{y}}_{m}(k \mid k-1)\right) \\
\hat{\mathbf{x}}(k+1 \mid k) & =A \hat{\mathbf{x}}(k \mid k)+\mathbf{B}_{u} \mathbf{u}(k)+\mathbf{B}_{v} v(k)
\end{aligned}
$$

By combining them, the overall state observer is given by

$$
\begin{aligned}
\hat{\mathbf{x}}(k+1 \mid k)=\left(\mathbf{A}-\mathbf{L} \mathbf{C}_{m}\right) \hat{\mathbf{x}}(k \mid k) & +\mathbf{L} \mathbf{y}_{m}(k)+\mathbf{B}_{u} \mathbf{u}(k) \\
& \times\left(\mathbf{B}_{v}-\mathbf{L D}_{v m}\right) v(k)
\end{aligned}
$$

where $\mathbf{L}=\mathbf{A M}$ is the observer gain.

\subsection{MPC results}

Figures 7 and 8 show the MPC performance to a $+10 \%$ increase in both the raw feed TOC and $\mathrm{NH}_{4}^{+}$concentrations. Real-time measurements of the RFT TOC are not available. Observe that the settling time for both the PB and TR reactors' effluent $\mathrm{pHs}$ is decreased and that the effluent compositions of PB and TR are well regulated about their set points (the deviation of TOC and $\mathrm{NH}_{4}^{+}$from their nominal values).

\section{TEST RESULTS AND ANALYSIS}

A supervisory control system failure scenario assuming the loss of TCP/IP connection is imposed on the testbed. As illustrated in Fig. 9, the normal mode with the supervisory controller 

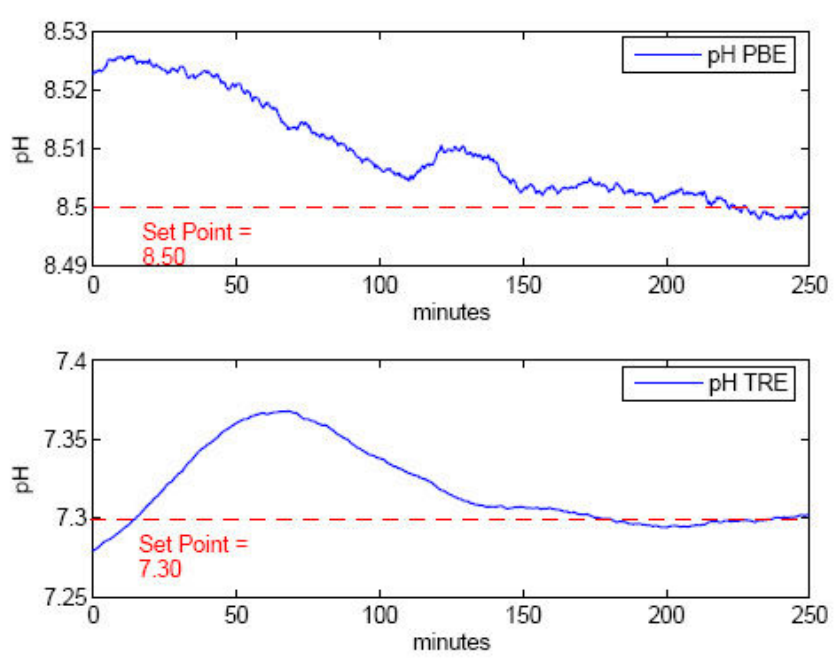

Fig. 7. $\mathrm{pH}$ regulation by MPC - testbed.
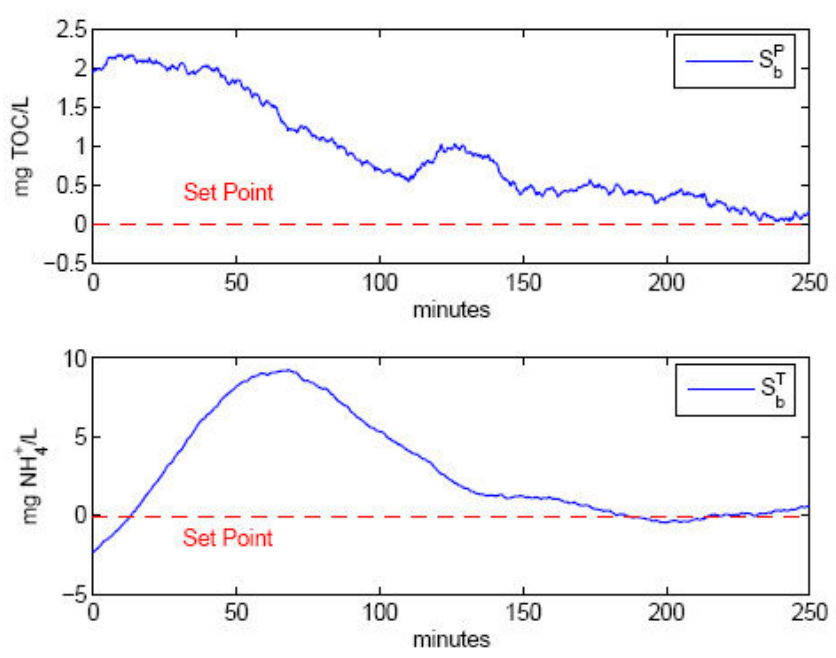

Fig. 8. Effluent concentration regulation by MPC - testbed.

online is operational up to $T_{F}=20$ minutes. At $T_{F}$, the data link is terminated, and the decision making logics generates the event signal, $F=1$. As a consequence, the finite state machine switches the hierarchical control system into the safety mode.
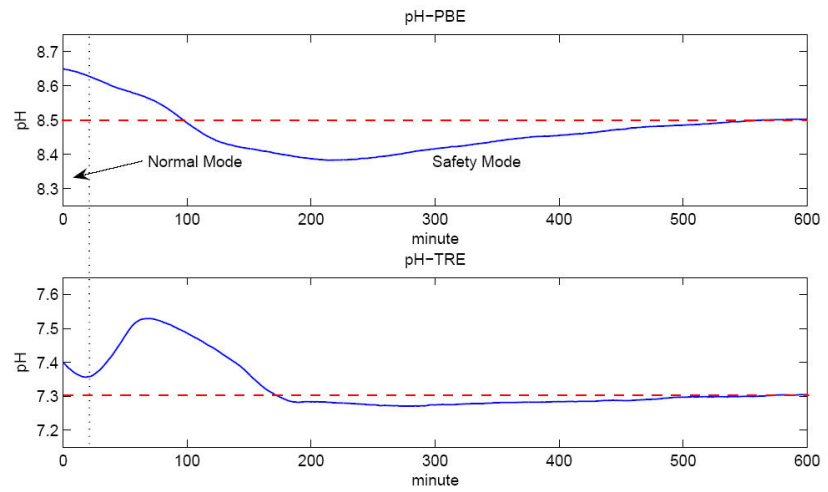

Fig. 9. Safety mode test.

From Fig. 9, it can be concluded that the safety mode is able to bring the process back to the desired operating condition (dashed line) after the loss of the supervisory controller. A drawback is that with the current tuning parameters, the controllers take too long to return the system to its set points (settling time is up to 600 minutes for the PB and 500 minutes for the TR). This is partly because the initial $\mathrm{pH}-\mathrm{PBE}$ deviation from the desired condition is large and also the local control scope of the two distributed embedded controllers. But the catastrophic failure of the biological plant is avoided by the embedded control configuration and the hierarchical control architecture.

\section{SUMMARY}

This work addressed the control of a coupled set of biological reactors used to treat yellow and grey water. A requirement of the control strategy is robustness and autonomy. To achieve some degree of autonomy a hierarchical control strategy that consisted of two embedded single-loop feedback controllers on one level and a centralized supervisory structure on the other was investigated. In the normal mode, the supervisory controller coordinates the behavior of the regulators, while in the safety mode, the embedded controllers operate autonomous of the supervisory controller. A finite state machine is used to achieve mode switching. Experimental results provided demonstrated the potential of the hierarchical control design.

\section{REFERENCES}

P. J. Antsaklis, K. M. Passino, and S. J. Wang. An introduction to autonomous control systems. IEEE Control System Magazine, 11:5-13, 1991.

A. Bemporad and M. Morari. Control of systems integrating logic, dynamics and constraints. Automatica, 35:407-427, 1999.

L.G. Bleris, J. Garcia, M.V. Kothare, and M.G. Arnold. Towards embedded model predictive control for system-on-achip applications. Journal of Process Control, 16:255-264, 2006.

C. Brosilow and B. Joseph. Techniques of Model-Based Control. Prentice Hall, Upper Saddle River, NJ, 2002.

W.A. Jackson. Stoichiometry of wrs. Internal report.

T. Kailath. Linear Systems. Prentice-Hall, Englewood Cliffs, NJ, 1980.

M. D. Lemmon, K. X. He, and I. Markovsky. Supervisory hybrid systems. IEEE Control Systems Magazine, 19:42-55, 1999.

D.T. Muirhead, T. Rector, W.A. Jackson, H. Keister, A. Morse, K. Rainwater, and K.D. Pickering. Performance of a small scale biological water recovery system. In ICES Proceedings. Soc. of Auto. Eng., 2003. \#2003-01-2557.

B.A. Ogunnaike and W.H. Ray. Process Dynamics, Modeling, and Control. Oxford University Press, NY, 1994.

B. Rittman and P. McCarty. Environmental Biotechnology. McGraw Hill Higher Education, NY, 2001.

C.E. Verostko, C. Carrier, and B.W. Finger. Ersatz wastewater formulations for testing water recovery systems. Soc. of Auto. Eng., 2004. \#2004-01-2448.

X. Zhang and K. A. Hoo. Controlling a coupled set of biological reactors for wastewater treatment. J Control Engineering Practice, 16:553-568, 2008.

X. Zhang, K.A. Hoo, and D. Overland. Kinetic-based modeling and feedback control studies of an integrated bio-reactor system. In Proc. 2006 American Control Conference, Minn., MN, June 2006. paper WeA05.1. 